\title{
El Castillo de La Vilavella (Castellón). Estudios y primeras actuaciones de conservación
}

The Castle of La Vilavella (Castellón). Surveys and first conservation actions

\author{
Camilla Mileto $^{\text {a }}$, Fernando Vegas ${ }^{\text {b }}$, Lidia García-Soriano ${ }^{c}$, Salvador Tomás Marquez ${ }^{\text {d }}$ \\ Universitat Politècnica de València, Valencia, Spain

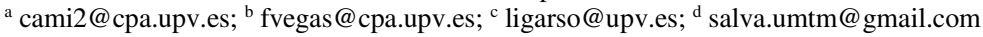

\begin{abstract}
The castle of La Vilavella (Castellón) stands on the slopes of San Sebastián, and its various constructions follow the craggy relief of the hill, on the outskirts of the town to the west. The castle is currently an imposing ruin on top of a hill, a landmark which dominates the inland landscape as well as the plains to the sea. Part of the castle's walled complex, which stretches along $230 \mathrm{~m}$ of the local topography with a maximum width of $60 \mathrm{~m}$, is conserved. The walled complex incorporates a sequence of towers of different types, forms, and constructive techniques which reflect the different periods in which the complex was built. This article aims to present the conservation project carried out in the castle, as well as the prior analyses and completed work based on these. The starting point for this conservation project was that the actions on a major heritage asset such as this constitute another phase for learning about its material history. They also make it possible to continue research into its material and constructive history through new archaeological excavations such as the study of its constructive elements. The interventions carried out aim to respect the construction of the castle at both a material and aesthetic level, returning it to a physical condition which honours its history and helps prolong its useful life. The conservation work carried out, necessary to ensure consolidation, has focused mostly on the walls of the different structures and adaptation and musealisation actions of the castle complex.
\end{abstract}

Keywords: Castle of La Vilavella, conservation project, rammed earth.

\section{Introducción}

El término de La Vilavella (Castellón) forma parte de la Comarca de la Plana Baja. La localidad se sitúa, más concretamente, al pie de una estribación de la Sierra de Espadán, a unos 38 m sobre el nivel del mar y aproximadamente a 7 $\mathrm{km}$ de la costa, con pendiente media del $0,5 \%$. Tiene una extensión aproximada de $6,1 \mathrm{~km}^{2}$, con una forma rectangular y una longitud máxima en el sentido este-oeste, que va ganando altura desde sus construcciones más recientes hasta el centro histórico, en la falda oeste del cerro de San

Sebastián, elevándose con mayor celeridad en este punto.

El castillo de La Vilavella se alza sobre el nombrado cerro de San Sebastián, adaptándose en sus diferentes construcciones a la orografía escarpada de la colina, al oeste y a las afueras de la localidad. Pese a las buenas condiciones estratégicas, defensivas, y de gran visibilidad, el acceso presenta diversas dificultades. Este se produce a través de una senda desde la carretera, en la ladera norte y noroeste (siendo la zona de mayor suavidad en la pendiente), mediante un camino 
estrecho, por tramos, que se adapta a las características del terreno. Así, el Castillo de La Vilavella se presenta actualmente como una imponente ruina en la cima de una colina, un hito en el paisaje que domina tierra adentro y la planicie hasta el mar.

\subsection{El castillo}

El Castillo de La Vilavella, también conocido como Antiguo Castillo de Nules, es un monumento catalogado como Bien de Interés Cultural, según consta con el número de identificación: RI-51-0011002 y fecha de anotación ministerial 18 de marzo de 2003.

La Vilavella actual tiene su origen en el asentamiento musulmán que los historiadores datan en torno a los siglos IX-X, estableciéndose sobre restos de antiguos asentamientos íberos y romanos, como demuestra el hallazgo de diversos restos y piezas de dichas épocas.

Es en época de dominación cristiana, entre los siglos XIII y XIV, cuando el castillo se reforma por completo y se amplía hasta completar su trazado definitivo. Así, se proyecta y construye un castillo con tres recintos: un área palacial, un recinto superior que la engloba y un recinto inferior o cerca. Debido al mal estado de conservación de los restos, resulta prácticamente imposible, sin una campaña arqueológica exhaustiva, afirmar con rotundidad cuál era la traza exacta del castillo, o incluso el número de torres con que contaba, sobre todo en el frente sur, cuyos lienzos apenas son visibles hoy por hoy. El castillo conserva parte de su recinto amurallado con un desarrollo alargado que se adapta a la topografía del lugar con unos $230 \mathrm{~m}$ de longitud y 60 $\mathrm{m}$ de anchura máximos. A lo largo del recinto amurallado se localiza una secuencia de torres de diferente tipo, forma y técnica constructiva que anticipan la variedad de las épocas constructivas en las que se construyó el conjunto.

\section{Estado de conservación previo a la inter- vención}

El estado de conservación del castillo de La Vilavella previo a la intervención era de avanzado estado de degradación, con una conservación deficiente dado el escasísimo mantenimiento del mismo. En la mayoría de los elementos las estructuras han permanecido en pie en continuo deterioro, lo que hacía imprescindible la actuación sobre las mismas para evitar su progresiva desaparición. Las características del bien lo convierten en patrimonio de gran interés, muy frágil frente a los agentes atmosféricos y patológicos. El escaso acondicionamiento, había convertido el espacio en una zona poco atractiva para el usuario, influyendo notablemente en la visitabilidad, y con ello en el abandono y la incapacidad de puesta en valor.

Esta situación suponía un problema notable en lo que respecta a los trabajos de conservación, ya que complica el traslado de materiales y herramientas necesarias para su ejecución. La naturaleza había crecido con libertad no sólo en el itinerario de acceso, sino también en la superficie de desarrollo del propio castillo, colmatando el recinto en el interior e impidiendo la observación y análisis pormenorizado de los diferentes elementos arquitectónicos. Era el resultado de siglos de acumulación de terreno y restos de las propias construcciones caídas. Este estado, además, dificultaba los trabajos de estudio ya que algunas estructuras estaban parcialmente enterradas, comprometiendo la comprensión del espacio, requiriendo de una intervención que se adaptase a lo visible para regenerar el conjunto ante la imposibilidad de acometer una excavación arqueológica global. Por otro lado, es posible suponer que estas estructuras enterradas probablemente mantienen un mejor estado de conservación general. Esto es observable, por ejemplo, en el caso de la cata realizada en 2010, en el frente noroeste, donde se aprecia una costra mejor conservada en el muro de tapia en correspondencia a la zona recientemente excavada.

Volumétricamente, el castillo presenta siete torres defensivas, a las que se les suman los torreones sudeste y noroeste y la torre avanzada también en esta misma orientación. Éstas muestran una altura muy variable de entre 4,5 y 8,5 m aproximadamente, altura alcanzada únicamente por parte de la estructura vertical portante. Las torres que presentaban un peor estado de conservación eran la del área palacial y la torre más occidental del lienzo de muralla norte, las cuales habían experimentado colapsos de sus bases de 
mampostería apoyadas sobre relleno del terreno, comprometiendo su integridad volumétrica y creando nuevas vías de degradación. Era especialmente preocupante el derrumbe completo de muros de tapia en algunos puntos, potenciando su aparente discontinuidad. El resto de las torres presentaban un estado actual similar entre sí, con mamposterías localmente desprendidas, coronaciones disgregadas sin ningún tipo de protección, y erosiones en las juntas de mortero. Cabe destacar la situación más específica de la torre localizada inmediatamente al sur del área palacial (torre 4), la cual presentaba una grieta notable en su frente suroeste, además de la pérdida de material y el colapso de gran parte de los muros. Junto a las torres se han conservado diversos lienzos de muralla, siendo el que presenta mejor estado de conservación el ubicado al norte del conjunto.

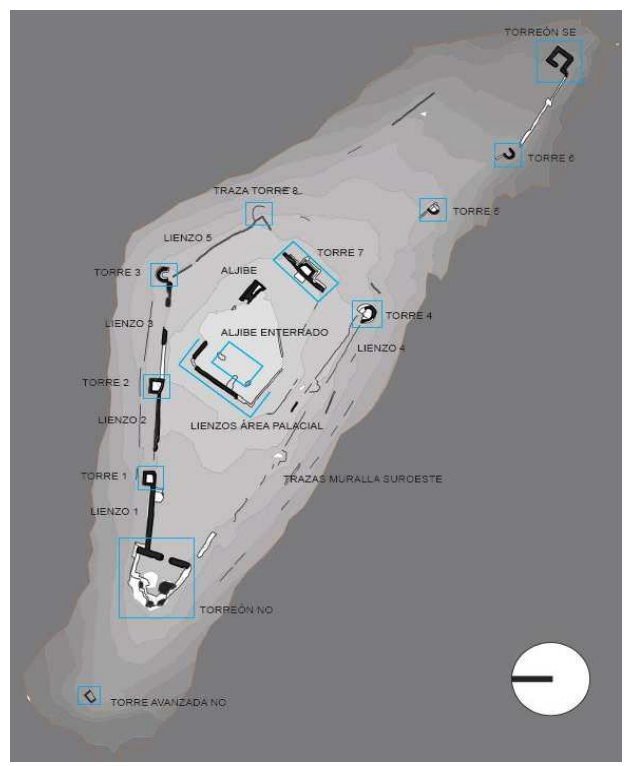

Fig. 1. Planta general del Castillo.

En estos lienzos se reconocen dos técnicas de tapia esenciales, las cuales presentan diferentes estados de avance de la degradación, de un espesor aproximado de 1,2 $\mathrm{m}$ y levantados sobre la superficie rocosa de la montaña.

En el caso de la tapia calicostrada, que es una técnica generadora de una superficie exterior con alto contenido en cal, capaz de conferir una pro- tección exterior en forma de costra, se presenta en muchas ocasiones semidescompuesta. La mayor resistencia que ofrece esta capa protectora frente al relleno de masa de tierra apisonada supone una pervivencia superior de esta, resultando en la aparición de oquedades de diferentes magnitudes según si han actuado con simultaneidad otros factores como desprendimiento de mampuestos de la base o una mala calidad de ejecución del paramento. Por otra parte, en el caso de la tapia careada con ladrillo (tapia valenciana), los ejemplares más afectados presentaban un estado heterogéneo. El refuerzo de ladrillo en la superficie exterior había permitido la mayor resistencia de estas zonas, observándose un aspecto similar al de la tapia calicostrada. Una de las localizaciones más críticas con respecto a este estado era la cara sur del lienzo norte del castillo, donde todo el centro de muro ya había caído, extendiéndose desde este punto hacia los extremos, y reconociéndose por ello una caída progresiva del relleno.

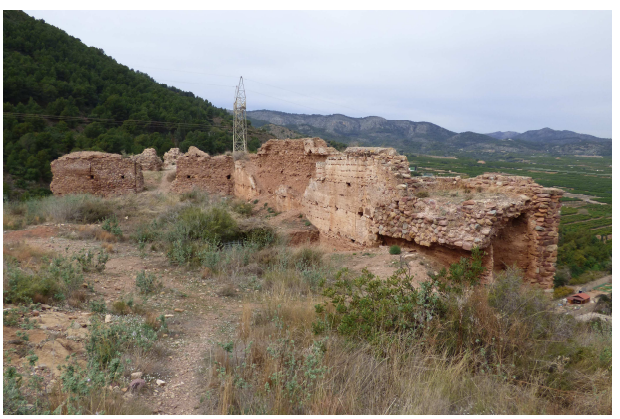

Fig. 2. Interior del recinto superior desde el área palacial. De frente los restos del torreón noroeste, y a la derecha, los de del lienzo 1 y torre 1 de la muralla norte.

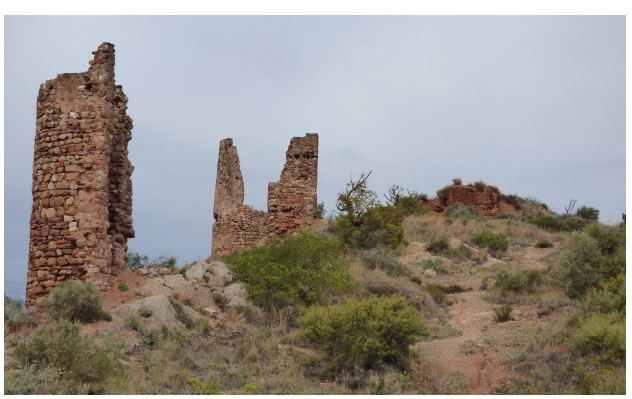

Fig. 3. Vista del recinto inferior desde el torreón sureste. Al fondo la torre 7; a la izquierda las torres 4,5 y 6 . 


\section{Elaboración de un plan de etapas}

En abril de 2018 se elaboró la redacción de un informe o Estudio Marco y Plan de Etapas, que se propuso como punto de partida para aglutinar los estudios realizados hasta la fecha, aporta una documentación del estado del conjunto y un plan de etapas para su estudio e intervención.

Puesto que no todas las construcciones presentaban el mismo nivel de degradación, el plan de etapas estableció distintos grados de urgencia de las intervenciones necesarias en base al riesgo para la integridad de la estructura por causa de los daños constatados.

Nivel de urgencia 1: Las actuaciones más urgentes de reparación / consolidación / estabilización de las áreas que presentan daños estructurales de mayor consideración. Se trata de los elementos y zonas que constituyen puntos críticos de cara a la conservación de la construcción, estando amenazada su integridad de no intervenirse sobre ellos. En este nivel se incluyeron el lienzo 1, los lienzos del área palacial, la torre $1 \mathrm{y}$ la torre 7.

Nivel de urgencia 2: Se establece como nivel de urgencia 2 el de las actuaciones de reparación / consolidación / estabilización de las áreas que presentan daños estructurales de consideración, pero en los que la inminencia de colapso o daño irreparable se prevé menor que en el caso anterior, sin dejar por ello de requerir una actuación urgente. En el castillo se detectó este nivel de urgencia en la torre 4 , lienzos 2,3 y 5 , en la torre 8 y en las trazas de la muralla suroeste.

Nivel de urgencia 3: Se establece como nivel de urgencia 3 el de las actuaciones de reparación / consolidación / estabilización de las áreas que presentan daños estructurales medios, en los que no se prevé un colapso inminente, sin dejar por ello de requerir una actuación a medio plazo que garantice este aspecto. En este nivel se incluyeron los torreones noroeste y sureste, las torres 3 y 5 , y el aljibe.

Nivel de urgencia 4: Se incluyen las actuaciones de reparación / consolidación / estabilización de las áreas que presentan daños no estructurales o daños estructurales leves, y en los que la intervención se entiende dirigida a mejorar el estado de mantenimiento. Los elementos incluidos en este nivel fueron la torre 2 y 6 , y el lienzo 4 .

Nivel de urgencia 5: Corresponde con las actuaciones relacionadas no ya con la propia construcción, sino con su puesta en valor (señalética, etc).

En este plan de etapas se realizaron una serie de fichas por elemento en las que se recogía una descripción general del mismo (con fotografías y plano de situación), un análisis de los materiales las técnicas constructivas que lo configuran, un estudio de la degradación material y/o estructural y una descripción del estado de conservación y de la necesidad de intervención.

\section{Proyecto de intervención. Criterios}

Dada la trascendencia histórica del castillo, se consideró de gran importancia resolver correctamente los problemas detectados empezando por las zonas que se había identificado de máxima prioridad en el Estudio Marco y Plan de Etapas. Para ello, se redactó un proyecto de intervención a finales de 2018 en el que se plantearon intervenciones de consolidación estructural y protección respetuosas con las características y valores esenciales del castillo. La intención principal consistía en limitar el avance del deterioro y eliminar en la medida de lo posible los riesgos de pérdida progresiva de las estructuras, además devolver al conjunto a un estado de decoro. Para ello se ha planteado una campaña de consolidación de todos los elementos en riesgo (de erosión, deslizamiento, derrumbe, desprendimiento, etc.), aunque a costa de no poder abarcar intervenciones integrales. En todos los casos, se ha recurrido a materiales locales, que han permitido una buena integración de las intervenciones. Estos materiales similares a los originales se han puesto en obra con técnicas constructivas análogas, sin introducir tecnología externa en el edificio, para que pueda continuar funcionando como tradicionalmente: con una determinada transpiración y permeabilidad, condiciones de flexibilidad, rigideces, peso o reversibilidad, quedando excluidos de la actuación, por tanto, los morteros de cemento y materiales de funcionamiento o impermeabilidad similar. La adecuación de los caminos que permiten visitar el castillo ha trata- 
do de preservar las estructuras histórica y favorecer su accesibilidad.

Se trata de intervenciones encaminadas a la conservación de los restos patrimoniales, por lo que ha primado el mantenimiento de estos, buscando dar solución al origen de las patologías que acrecientan la pérdida del bien, de carácter frágil y no renovable. La intervención propuesta se ha planteado como una actuación sostenible en el amplio sentido de la palabra. Por una parte, a nivel material los trabajos se han planteado con materiales tradicionales en la medida de lo posible. El empleo de tierra local, además, se adapta a las condiciones medioambientales y geológicas de la localidad, y no supone grandes costes de desplazamiento o transporte. Se ha tratado de devolver el edificio a un estado de seguridad con las intervenciones mínimas necesarias a tal fin. A través de estos criterios, la restauración del castillo supone un beneficio evidente para el conjunto en sí, puesto que es el modo de asegurar la pervivencia del mismo, manteniendo y recuperando las huellas del paso del tiempo, pero también actualizándolo en lo imprescindible para permitir su mantenimiento en el siglo XXI.

\section{Actuaciones realizadas}

Con carácter previo a las intervenciones propias en los elementos construidos, se ha realizado la limpieza y desescombro de terreno con la supervisión de arqueólogo, en el interior del castillo, así como en aquellas zonas necesarias para el acceso al mismo o la instalación de los andamios y otros medios auxiliares. Se han seleccionado, cribado y acopiado tanto mampuestos, ripios como gravas originales, de forma que han podido ser reutilizados en la presente intervención. Con respecto a la vegetación, se ha realizado un desbroce de arbustos y hierbas. En las estructuras históricas, la vegetación se ha retirado de forma manual y especialmente atenta, a fin de evitar causar daños al arrancar las raíces. Posteriormente se ha aplicado biocida para minimizar el riesgo de reaparición de nueva vegetación.

\subsection{Actuación en el lienzo 1}

La actuación en el lienzo 1, referente a la tapia valenciana, ha consistido en la reintegración vo- lumétrica del mismo con la construcción de una nueva tapia encofrada a una cara. Para ello se ha realizado una tapia careada con ladrillo reutilizado con tongadas y costra de cal realizada con un mortero de cal hidráulica NHL-3 y árido de características análogas al original. La masa de la tapia se ha realizado con tierra proveniente de los alrededores y los ladrillos empleados en la costra son ladrillos históricos reutilizados.

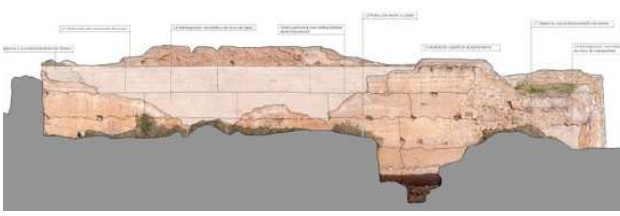

Fig. 4. Alzado de la actuación en el lienzo 1.

La conexión de la parte reintegrada del muro con el preexistente se ha realizado con una serie de estacas de madera ancladas en el cuerpo estable, con pasta de yeso en el retacado de la estaquilla y varillas de fibra de vidrio. La reintegración de esta gran laguna se ha dejado evidente y distinguible a través de un pequeño retranqueo que se genera entre la parte histórica y la parte de reintegración creado por el mismo desplome de la parte preexistente. Por otro lado, se ha perseguido la integración visual de la laguna mediante el cepillado de la costra de cal para obtener una textura rugosa similar a la costra histórica deteriorada y la integración cromática realizada con pátina de tierras naturales.

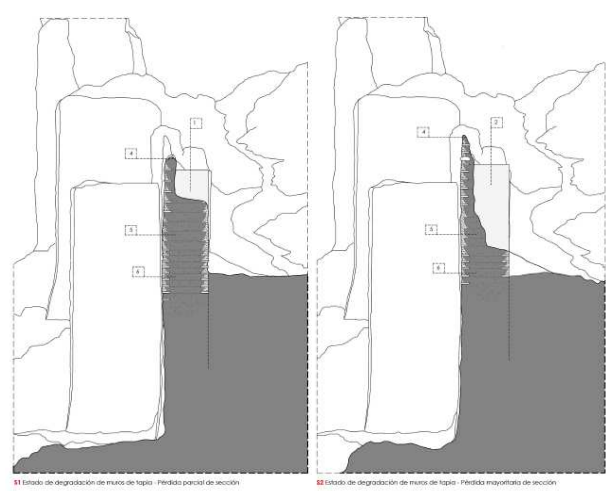

Fig. 5. Secciones de la actuación en el lienzo 1. 

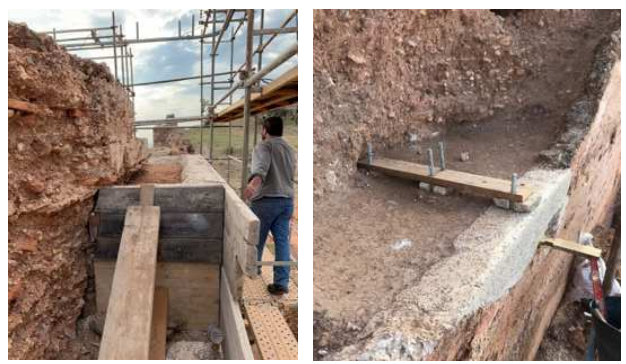

Fig. 6. Construcción de nueva tapia valenciana encofrada a una cara en el lienzo 1.

\subsection{Actuación en la torre 1}

En la torre 1 el núcleo de tierra del muro se había quedado expuesto tras el desprendimiento del paramento de mampostería.

Por ello, se ha realizado la reintegración de dicho paramento mediante la colocación de mampuestos de piedra caliza similares a las originales tomados con mortero de cal hidráulica NHL-3 y árido de características análogas al original cribado de las tierras excavadas del castillo.

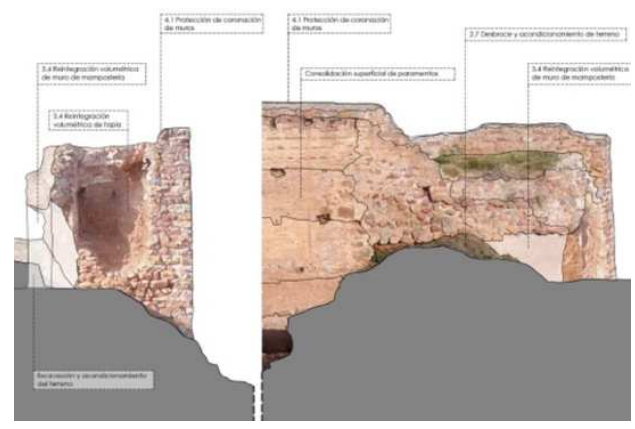

Fig. 7. Proyecto de la actuación en la torre 1.
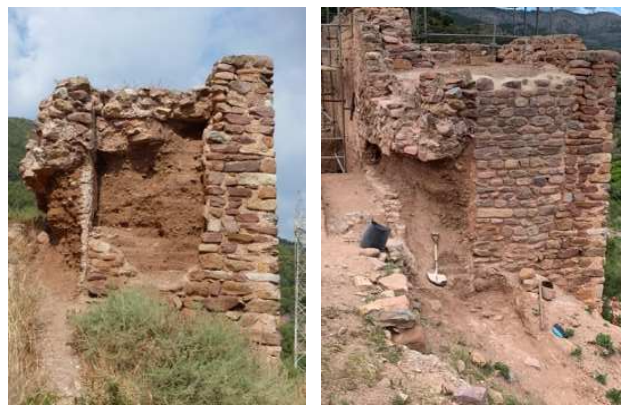

Fig. 8. Torre 1. Izquierda: imagen previa a la intervención, con el muro degradado. Derecha: imagen durante la intervención.
Con la misma técnica, se reintegró también el zócalo, en parte perdido de la cara meridional de la torre. Además de estas reintegraciones, se ha realizado la limpieza de la coronación de la torre tras la debida excavación arqueológica.

\subsection{Actuación en la torre 2}

La actuación en la torre 2 ha comprendido la limpieza de la coronación, la excavación arqueológica hasta encontrar el nivel del piso superior y el recrecido del antepecho de la cara oriental. Este recrecido se ha ejecutado siguiendo el mismo criterio que en el resto de las reintegraciones de los muros de mampostería, con piedra caliza similar a las originales tomadas con mortero de cal hidráulica NHL-3.

\subsection{Actuación en las torres $3,4,5$ y 6}

En las torres 3, 4, 5 y 6, con planta en forma de herradura, se ha actuado con un criterio de consolidación estructural y prevención de futuras pérdidas de material: en la coronación, se ha realizado la consolidación mediante inserciones puntuales de mampuestos y morteros de cal o con solo mortero de cal para mejorar su protección; en la base de los muros, se han hecho restituciones puntuales para asegurar su estabilidad; en las caras contra el terreno, se han consolidado los muros de contención del mismo.

\subsection{Actuación en la torre 7}

La torre 7 está construida con tapia calicostrada sobre un zócalo de mampostería. La actuación realizada en esta torre 7 ha tratado de consolidar los puntos más críticos estructuralmente, pero no se ha podido realizar una actuación integral puesto que este elemento necesita de una investigación mayor y de un mayor presupuesto para su correcta consolidación.

En esta fase del trabajo se ha consolidado una parte del lienzo de muralla adyacente a la torre y se han realizado reintegraciones puntuales en la misma, siguiendo los criterios expuestos en el apartado anterior. Así, se han reintegrado faltas puntuales y la esquina noroeste, que se había perdido casi íntegramente. 
Respecto a la coronación, por el momento no ha sido posible realizar tareas de excavación, limpieza o consolidación en la misma y se ha optado por protegerla con una capa geotextil cubierta con grava, esperando poder actuar sobre esta parte en un futuro próximo.
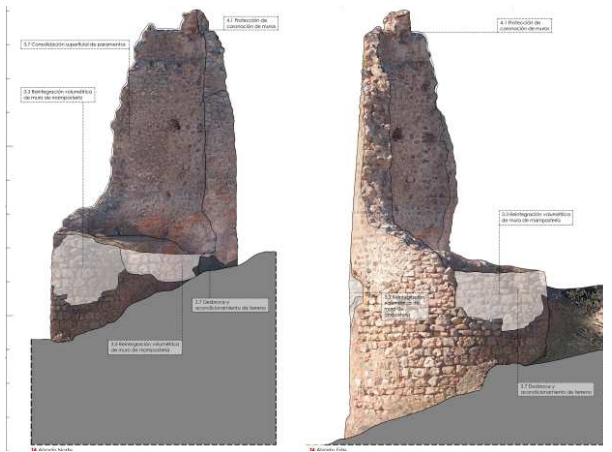

Fig. 9. Proyecto de la actuación en la torre 4.
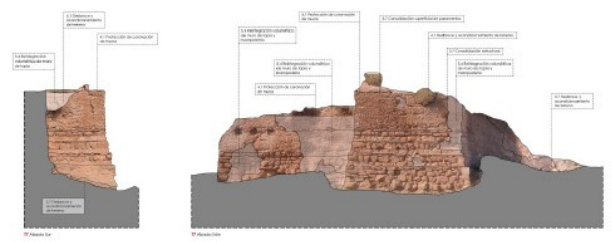

Fig. 10. Proyecto de actuación en la torre 7.
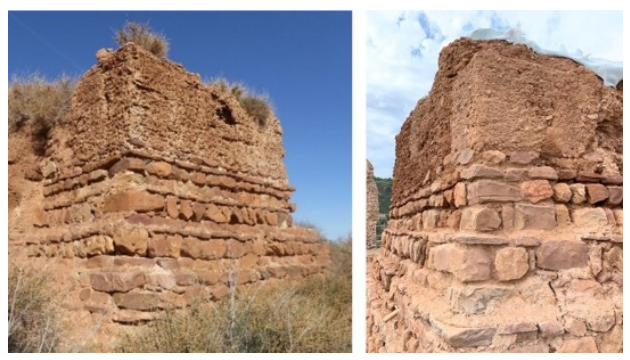

Fig. 11. Torre 7. Izquierda: imagen previa a la intervención. Derecha: reconstrucción de la esquina noroeste.

\section{Conclusiones}

En esta primera fase de intervención se ha podido actuar sobre aquellos elementos del castillo que requerían intervenciones más urgentes. No obstante, los trabajos en el Castillo de La Vilavella deberán seguir realizándose en el futuro a corto plazo para seguir interviniendo en los ele- mentos que no han podido ser objeto de actuación en este proyecto. En el caso concreto de la torre 7 será necesario realizar un estudio en detalle sobre la misma. Actualmente ya se han realizado algunos análisis (Alejandre Sánchez, et al., 2010) en los que se tomaron muestras de la costra, que fueron estudiadas mediante fluorescencia de rayos $\mathrm{X}$ y difracción por rayos $\mathrm{X}$, se determinaron los carbonatos de las mismas, se llevó a cabo un análisis mineralógico, un estudio de la granulometría en cada caso, se determinaron la densidad real y la aparente, así como el porcentaje de porosidad abierta y la resistencia mecánica a compresión. Estos análisis permitieron concluir, entre otras cuestiones, que las costras de las construcciones de tapia calicostrada analizadas están construidas con morteros de cal con dosificación cal:arena entre 1:3 y 1:2 (Mileto, et al., 2014).

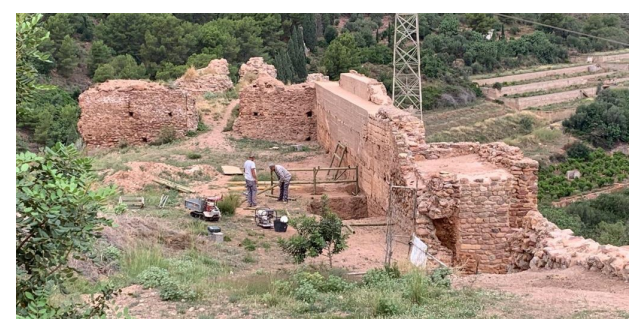

Fig. 12. Interior del recinto superior desde el área palacial. Imagen de la intervención del lienzo 1 y torre 1 de la muralla norte.

Por otro lado, es posible presuponer que gran parte de las estructuras del castillo se encuentran actualmente bajo el nivel del terreno. En el año 2010 los arqueólogos García Fuertes y Moraño Poblador llevaron a cabo un estudio del castillo que incluyó la excavación de catas que les permitieron un conocimiento más completo de las áreas excavadas (García Fuertes, Moraño Poblador, 2013) y plantean la existencia de tres fases constructivas: una primera que incluye la formación de un único recinto de planta indeterminada con torres cuadrangulares de mampostería, si bien no descartan que el recinto fuera poligonal; una segunda etapa relacionada con los elementos de tapia mixta, tapia calicostrada y torres de mampostería que aprovecha las trazas de la etapa anterior pero ejecutando un nuevo proyecto, incluida la construcción del recinto inferior; una 
tercera y última fase correspondiente a las diversas remodelaciones en los diversos frentes murarios, incluyendo la construcción del reducto en la torre noroeste. Por todo ello, será de vital importancia acompañar los futuros proyectos de actuaciones de excavación arqueológica que permitirán descubrir estas estructuras, o hacerlas accesibles, como es el caso de los aljibes, y que por supuesto, contribuirán a la puesta en valor de este elemento patrimonial. Así, los restos de esta fortificación se revelan como un yacimiento arqueológico de gran potencial, puesto que permanece inexcavada la práctica totalidad del recinto. El valor arqueológico del enclave radica no sólo en las estructuras existentes sino también en aquellas que puedan aflorar como fruto de las excavaciones arqueológicas. Dada la magnitud de la empresa, se plantea la necesidad de enfrentarla por etapas en campañas sucesivas que permitan, finalmente, un conocimiento lo más exhaustivo posible de un bien de gran importancia largamente olvidado. Estas campañas de excavación deben ir acompañadas siempre por los co- rrespondientes trabajos de consolidación de modo que no se degraden los restos descubiertos.

\section{Notas}

El proyecto de intervención en el Castillo de La Vilavella ha sido realizado por F. Vegas y C. Mileto. Los levantamientos del castillo se han llevado a cabo por P. Rodríguez Navarro y M. T. Gil Piqueras. La excavación arqueológica ha sido realizada en diversas fases por los arqueólogos J.M. García Fuertes, I. Moraño Poblador. La obra ha sido realizada bajo la dirección facultativa de los arquitectos C. Mileto y F. Vegas y el arquitecto técnico S. Tomás y la colaboración de L. García. El estudio y la intervención en este castillo forman parte de la investigación del proyecto "Risk-Terra. La arquitectura de tierra en la península ibérica: estudio de los riesgos naturales, sociales y antrópicos y estrategias de intervención e incremento de la resiliencia" concedido por Ministerio de Ciencia, Innovación y Universidades (ref. RTI2018-095302-B-I00).

\section{Bibliography}

Alejandre Sánchez, F.J.; Martín del Río, J.J.; Blasco López, F.J. (2010). Caracterización y elaboración de informe científico técnico de muestras de tapiales de las murallas del castillo de la Villavieja (Castellón), Inédito.

Felip, V.; García Edo, V. (2003). Privilegios y Concesiones del Término General del Castillo de Nules en Época Foral (1251-1709), Ayuntamiento de Nules.

García Fuertes, J.M.; Moraño Poblador, I. (2013). "El castell de La Vilavella: avance de los resultados obtenidos en la Campaña 2010 (La Vilavella, Castellón)”, Quaderns de Prehistòria i Arqueologia de Castelló, 31, pp. 255276.

Mileto, C.; Vegas, F.; Alejandre, F.J.; Martín, J.J.; García Soriano, L. (2014). "Lime-crusted rammed earth: materials study", Advanced Materials Research, 831, pp. 9-13.

Vegas, F.; Mileto, C. (2018). Estudio marco y plan de etapas del castillo de La Vilavella (Castelló). 